\title{
Numerical Simulation on Characteristics of Debris Clouds Produced by Conical Projectiles Hypervelocity Impact on Thin Plates
}

\author{
Xun $\mathrm{Liu}^{1 *}$, Fangfang $\mathrm{Gai}^{2}$ and Su Cheng ${ }^{1}$ \\ ${ }^{1}$ College of Civil Engineering and Architecture, Harbin University of Science and \\ Technology, Harbin 150080, China \\ ${ }^{2}$ College of Science, Heilongjiang Institute of Science and Technology, Harbin \\ 150022, China \\ liuxun0627@163.com
}

\begin{abstract}
With the unceasing development of space activity, the total number of space debris is ever increasing, which greatly threatens orbiting space vehicles. To research the characteristics of debris clouds which are produced by conical projectiles hypervelocity impact on thin plates, SPH (smoothed particle hydrodynamics) methods in AUTODYN is used. Characteristics of debris cloud produced by conical projectiles with different ratio between length and diameter hypervelocity impact on thin plates are investigated with the same mass and velocity by numerical simulation. Effects of the ratio between length and diameter of the projectiles affecting on the form of debris cloud, axial location of debris clouds, radial extension of debris cloud and tip particle velocity of debris cloud are obtained. It is shown that the degree of fragmentation of the projectiles and debris cloud diffusion is changed with the velocity of the projectiles, the ratio between length and diameter and the impacting direction. The increasing ratio between length and diameter of projectiles can reduce the damage of thin plates but rise the damage of the wall of spacecraft by the debris clouds impact on with the same mass and velocity.
\end{abstract}

Keywords: debris cloud, hypervelocity impact, conical projectiles, numerical simulation

\section{Introduction}

With the increasing of human space activities, space environment is becoming increasingly complex, space debris is one of the main threats to spacecraft [1]. At present, spherical projectiles have been the conventional shape used in most of the experiments and researches about the impact by space debris [2], while the shapes of space debris is various. Studies show that the impact by non spherical projectiles can cause more damage than by spherical projectile under the same conditions and the same quality [3-5], thus it is very important to use different shape of the projectiles for research the influence of the hypervelocity impact process.

The numerical simulation is an effective method to study the properties of protective structure on hypervelocity impact [6]. In this paper, the effect of conical projectiles with different ratio of length to diameter (abbreviated as L/D in this paper) and an impact orientation (face-on impact and point-on impact) is to be investigated. The characteristic of debris clouds is investigated by numerical simulation with AUTODYN-2D software. 


\section{Numerical Simulation}

According to the axial symmetry of the geometry of projectiles, using the nonlinear dynamics analysis software AUTODYN-2D, the calculation model of hypervelocity impact on thin aluminum plate by conical projectiles is established by using SPH method. Figure 1 shows the initial geometric model (due to axial symmetry, only half of the projectile and plate is established). All projectiles are made of Al-1010 aluminum alloy and have the same mass, 316mg. The plate is made of Al-2024 aluminum alloy with $1 \mathrm{~mm}$ thickness. The projectiles and the plate are using SPH modeling method and the particle diameter is $0.1 \mathrm{~mm}$. The state equation of material is the Shock state equation which provided by AUTODYN software database. The strength model adopts the Johnson-Cook model. In order to ensure the most impact body material is solid state, the initial impact velocity is set of $5 \mathrm{~km} / \mathrm{s}$.

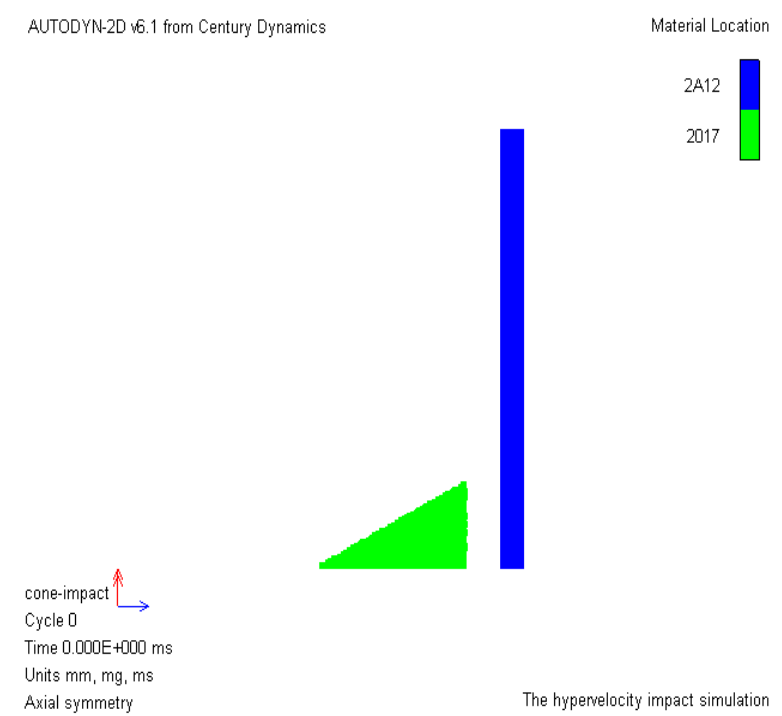

a) Face-on Impact

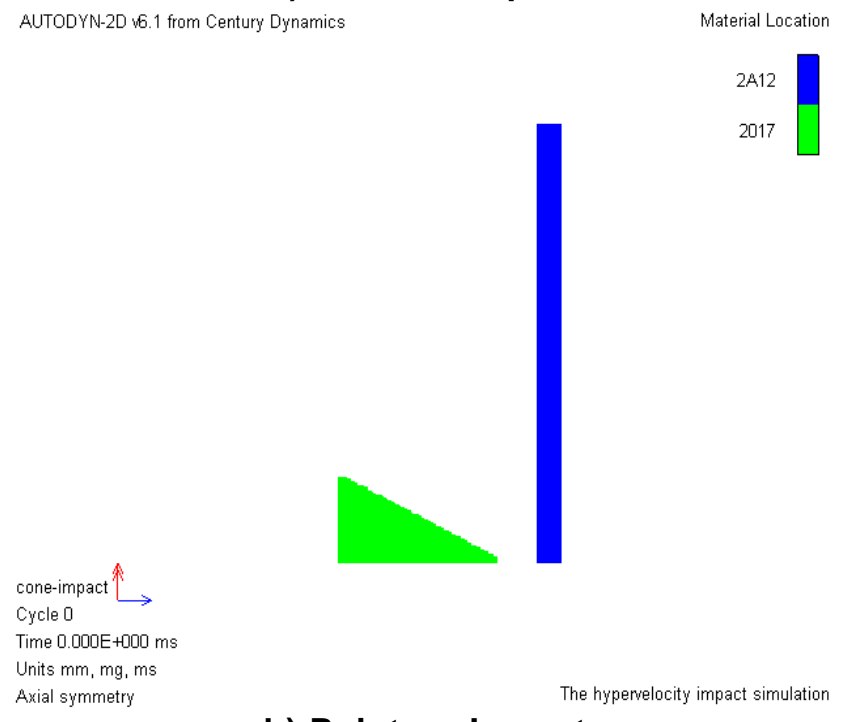

b) Point-on Impact

Figure 1. Initial Geometric Model

In order to research the effect of conical projectiles with different L/D and impact orientations on debris cloud characteristics, select 26 groups of cases of numerical 
simulation. The L/D of the projectiles is from 0.1 to 10 . The orientation and the L/D of each projectile are illustrated in Table 1.

Table 1. List of Cases of Numerical Simulation of Conical Projectiles

\begin{tabular}{cccc|cccc}
\hline \multirow{2}{*}{$\begin{array}{c}\text { Simulation } \\
\text { No. }\end{array}$} & Lace-on impact & L $(\mathrm{mm})$ & $\mathrm{D}(\mathrm{mm})$ & $\begin{array}{c}\text { Simulation } \\
\text { No. }\end{array}$ & $\mathrm{L} / \mathrm{D}$ & $\mathrm{L}(\mathrm{mm})$ & $\mathrm{D}(\mathrm{mm})$ \\
\hline 1 & 0.1 & 1.6287 & 1.6287 & 1 & 0.1 & 1.6287 & 1.6287 \\
2 & 0.2 & 2.586 & 12.93 & 2 & 0.2 & 2.586 & 12.93 \\
3 & 0.3 & 3.387 & 11.29 & 3 & 0.3 & 3.387 & 11.29 \\
4 & 0.4 & 4.104 & 10.26 & 4 & 0.4 & 4.104 & 10.26 \\
5 & 0.6 & 5.3778 & 8.965 & 5 & 0.6 & 5.3778 & 8.965 \\
6 & 0.8 & 6.5144 & 8.143 & 6 & 0.8 & 6.5144 & 8.143 \\
7 & 1.0 & 7.56 & 7.56 & 7 & 1.0 & 7.56 & 7.56 \\
8 & 2.0 & 12 & 6 & 8 & 2.0 & 12 & 6 \\
9 & 3.0 & 15.72 & 5.24 & 9 & 3.0 & 15.72 & 5.24 \\
10 & 4.0 & 19.04 & 4.76 & 10 & 4.0 & 19.04 & 4.76 \\
11 & 6.0 & 24.96 & 4.16 & 11 & 6.0 & 24.96 & 4.16 \\
12 & 8.0 & 30.24 & 3.78 & 12 & 8.0 & 30.24 & 3.78 \\
13 & 10 & 35.1 & 3.51 & 13 & 10 & 35.1 & 3.51 \\
\hline
\end{tabular}

\section{The Numerical Simulation Results and Analysis}

\subsection{Analysis of Shape of Debris Cloud}

Figure 2 shows the numerical simulation results of debris clouds generating from conical projectiles with different $\mathrm{L} / \mathrm{D}$ and different impact orientation at the time of $15 \mu \mathrm{s}$ after impact. Further analysis shows that when L/D reaches 0.6 and 2 at face-on impact, or when L/D reaches 0.6 and 4 at point-on impact, the shape of the debris cloud changes great, and when the L/D of the projectiles is different, the degree of fragmentation of the projectiles are also different.

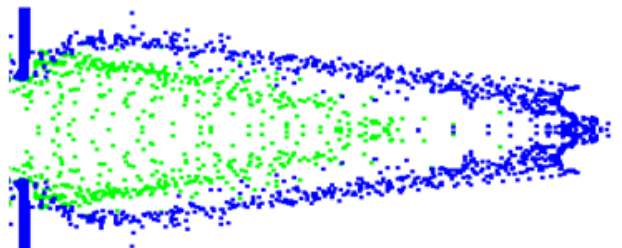

face-on impact

a) $L / D=0.1$

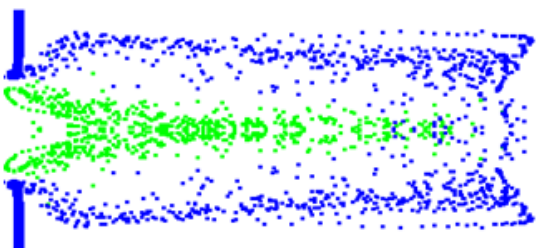

point-on impact

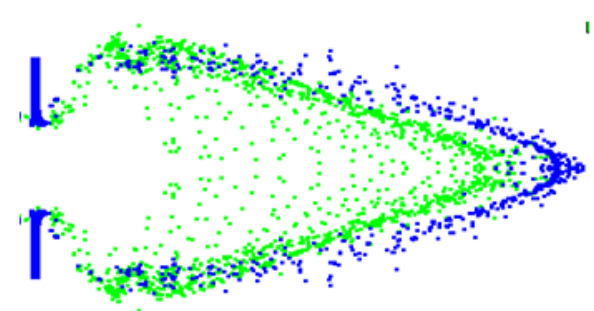

face-on impact

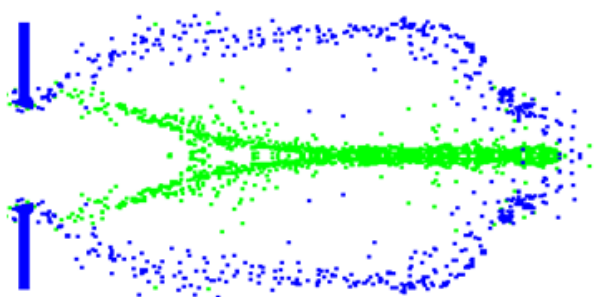

point-on impact

b) $L / D=0.3$ 


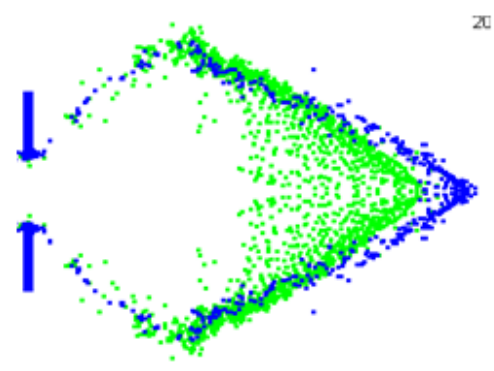

face-on impact

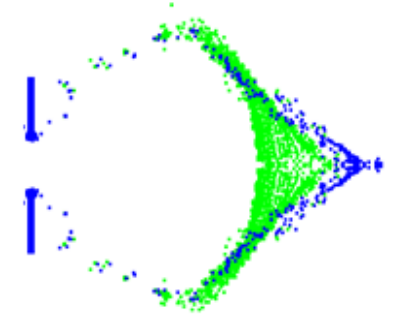

face-on impact

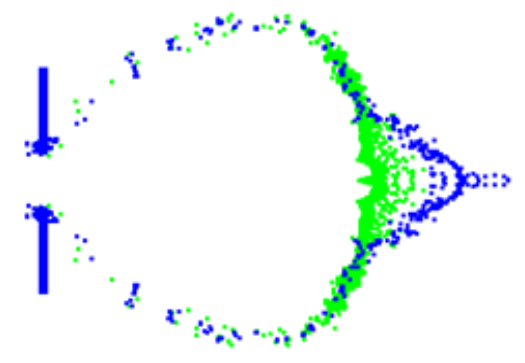

face-on impact

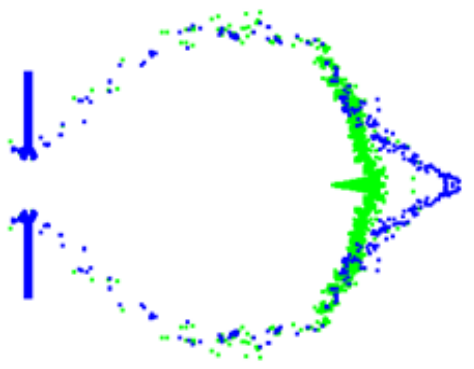

face-on impact

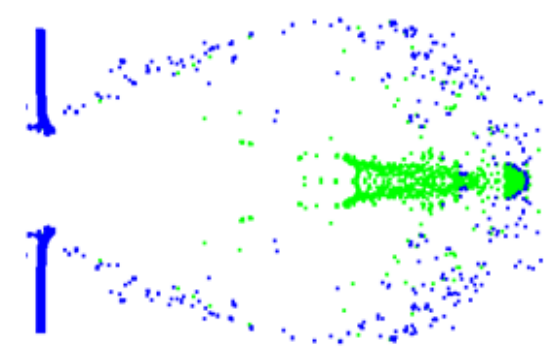

point-on impact

c) $L / D=0.6$

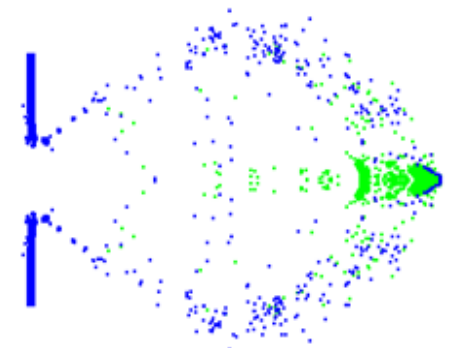

point-on impact

d) $L / D=1$

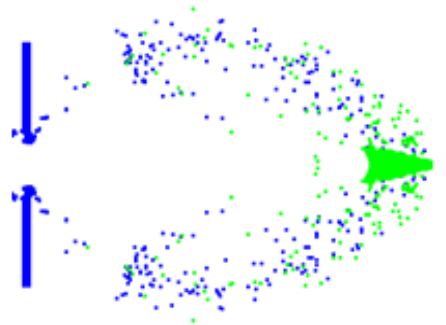

point-on impact

e) $L / D=2$

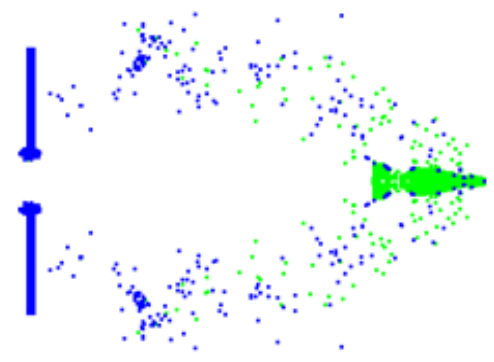

point-on impact

f) $L / D=3$ 


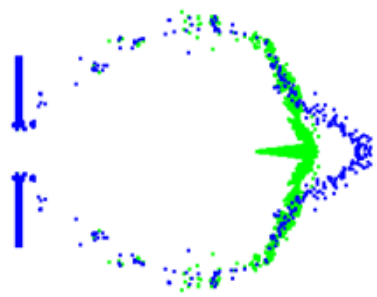

face-on impact

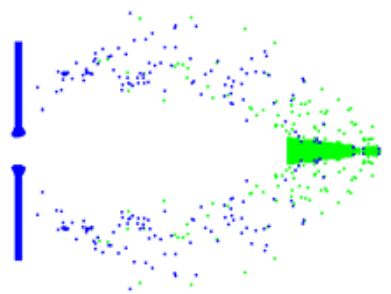

point-on impact

g) $L / D=4$

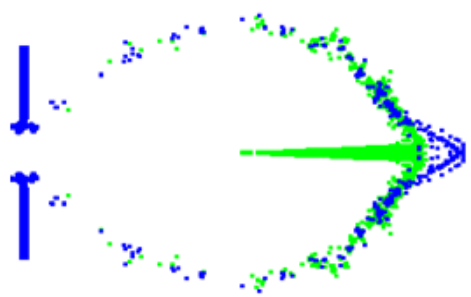

face-on impact

h) $L / D=10$

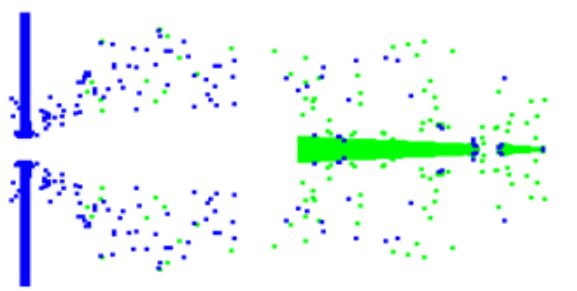

point-on impact

\section{Figure 2. Numerical Simulation Results of Debris Clouds Generating From Conical Projectiles with Different L/D and Different Impact Orientation}

For face-on impact, the shape of the debris cloud is conical when L/D is no more than 0.6 , and there is no columnar structure in it, in the front of the debris cloud there exists concentrated debris, when L/D is between 0.6 and 2, there are concentrated debris in the middle and in the front of the debris clouds, when L/D is reaches 2 or over, the front of the projectile is fragmentized, but the back the projectile is not fragmentized, maintaining the original shape, located in the axis of the projectile.

For point-on impact, the shape of the debris cloud is cylindrical when L/D is no more than 0.6, there is a columnar structure generated by the concentrated fragments in the debris cloud, and there exists bifurcation at the back end, when L/D is between 0.6 and 4, the debris cloud is concentrated, mainly located in the axis of the projectile, when L/D is reaches 4 or over, the front of the projectile is fragmentized, while the back the projectile is not fragmentized, maintaining the original shape, located in the axis of the projectile.

In summary, when the velocity and quality of the conical projectiles is the same, the different $\mathrm{L} / \mathrm{D}$ of the projectile and impact orientation will lead to a great change in the shape of debris cloud. The larger the L/D of the projectiles cause the more concentrated quality of the debris cloud.

\subsection{Analysis and Comparison of Debris Clouds Parameters}

For further analysis of the influence of the debris cloud generating from conical projectile by different L/D and impact orientation, select the front tip velocity of the debris cloud, diameter of radial expansion of the debris cloud and the perforation diameter to analyze.

Figure 3- Figure 5 show the relationship between the front tip velocity of the debris cloud, diameter of radial expansion of the debris cloud, the perforation diameter and the $\mathrm{L} / \mathrm{D}$ of the projectiles respectively at the time of $15 \mu \mathrm{s}$ after impact.

Figure 3 shows that the L/D of projectile has obvious effect to the front tip velocity of the debris cloud when the projectile face-on impact on the plate, but has almost no effect to that when the projectile point-on impact on the plate. 
Figure 4 shows that the diameter of radial expansion of the debris cloud is increasing with the increase of the L/D when the L/D is less than 1, but when the L/D is more than 2, the diameter of radial expansion of the debris cloud is decreasing with the increase of the $\mathrm{L} / \mathrm{D}$, hence the diameter of radial expansion of the debris cloud has maximum size when the L/D is between 1 and 2. It also shows that the diameter of radial expansion of the debris cloud when projectile face-on impact on the plate is larger than that when the projectile point-on impact on the plate. In summary, with the increase of the L/D, the kinetic energy of the debris cloud is increased, and cause more damage to the bulkhead by the debris cloud.

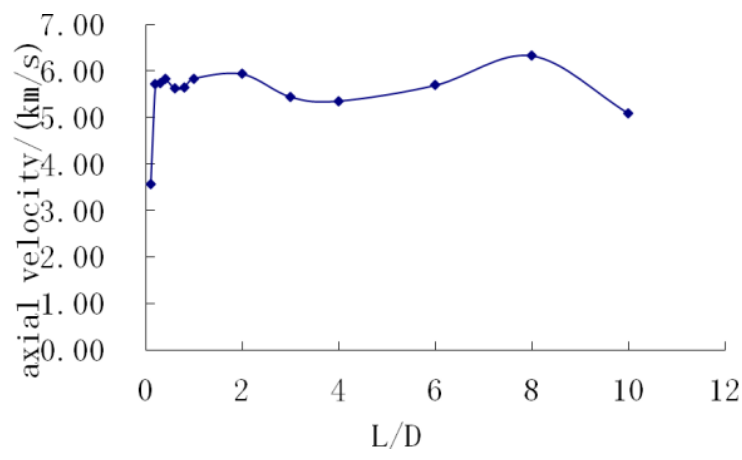

a) face-on Impact

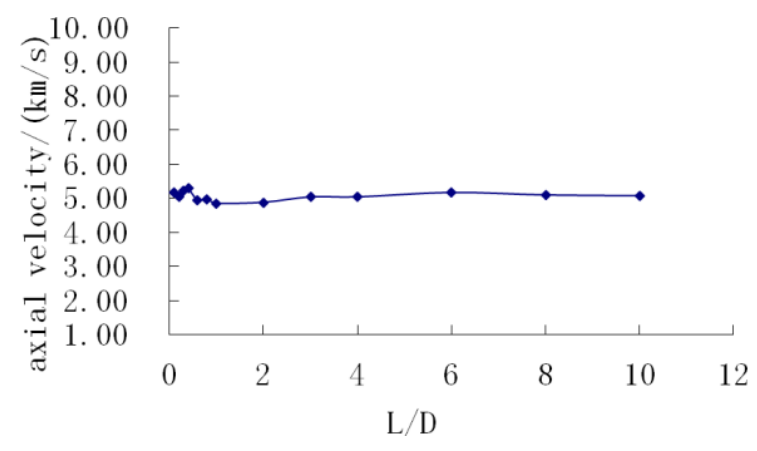

b) point-on Impact

Figure 3. Front Tip Velocity of Debris Cloud

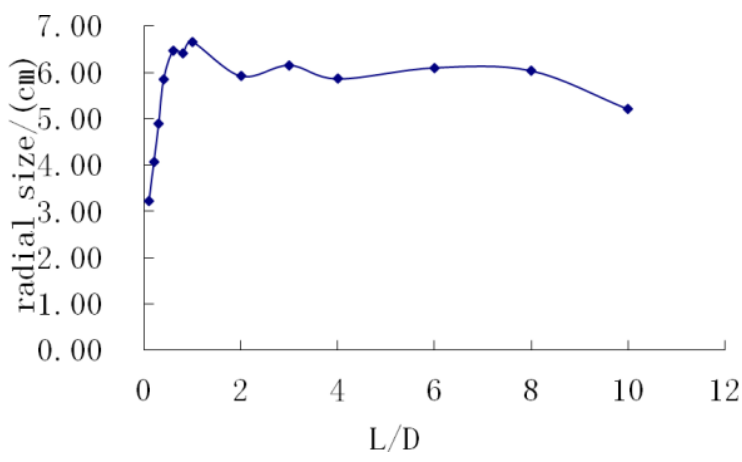

a) face-on Impact 


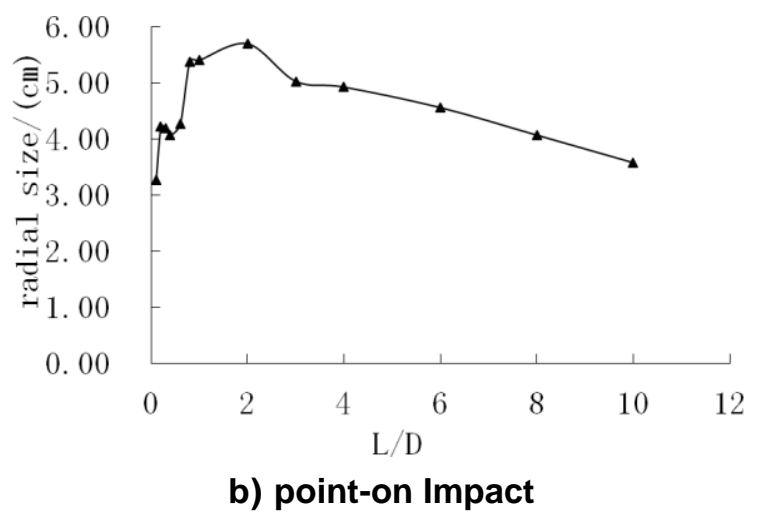

Figure 4. Diameter of Radial Expansion of Debris Cloud

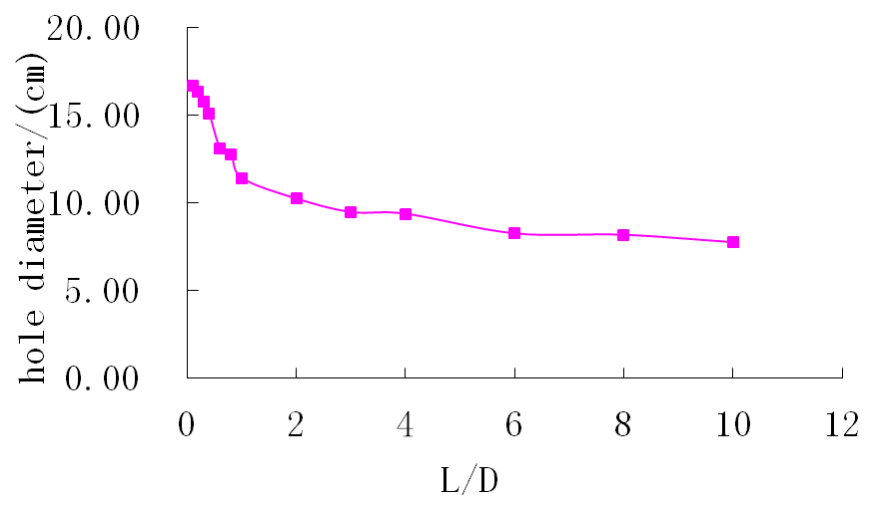

a) face-on Impact

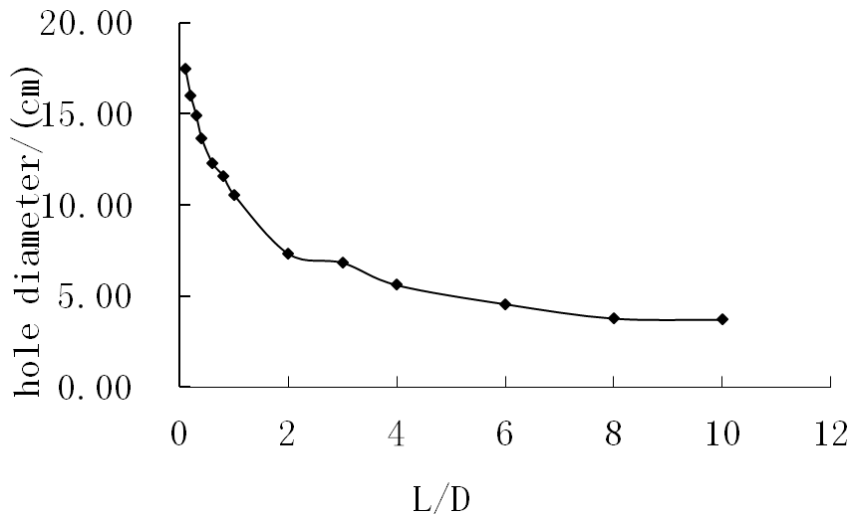

b) point-on Impact

Figure 5. Perforation Diameter

Figure 5 shows that the perforation diameter is decreasing with the increase of the L/D of the projectile, and it decreases more when the projectile point-on impact on the plate than when the projectile face-on impact on the plate.

\section{Conclusion}

Numerical simulations by AUTODYN-2D have been performed for the impact of conical projectiles on thin plate to investigate the characteristics of the debris clouds with SPH method applied. The effect to shape of debris cloud and motion parameters by 
conical projectiles with different $\mathrm{L} / \mathrm{D}$ and impact orientations is analyzed on the condition of same mass and initial impact velocity of the projectiles. The results show:

1) The larger the ratio of length to diameter (L/D) of the projectiles be, the more the quality of the debris cloud concentrated, and when the projectile face-on impact and the $\mathrm{L} / \mathrm{D}$ is reach to 2 or over, or when the projectile point-on impact and the $\mathrm{L} / \mathrm{D}$ is reach to 4 or over, the projectile has residual length after impact, furthermore, with the L/D of projectile increases, the residual length of the projectile increased.

2) The increase of $L / D$ of the projectile can reduce the damage of the thin plate, hence it can increase the damage of the external bulkhead of spacecraft.

3) Compare the two different impact orientations, for the effect of the characteristic parameters of debris cloud, the changes of $\mathrm{L} / \mathrm{D}$ when the projectile face-on impact on the plate cause more influence than that when the projectile point-on impact on the plate, while for the effect of the damage of the thin plate, the changes of L/D when the projectile point-on impact on the plate cause more influence than that when the projectile face-on impact on the plate.

The results of the paper can provide reference for the spacecraft hypervelocity impact risk assessment and for protection engineering design.

\section{Acknowledgements}

This work is supported by National Natural Science Foundation of China (11202070). Computer time support was provided by the Hypervelocity Impact Research Center at the Harbin Institute of Technology.

\section{References}

[1] N. N. Smimov, A. B. Kiselev and K. A. Kondratyev, "Impact of Debris Particles on Space Structures Modeling", Acta Astronautica, vol. 67, (2010), pp. 333-343.

[2] G. A. Graham, A. T. Kearsley and M. M. Grady, "Natural and simulated hypervelocity impacts into solar cells", Int. J. Impact Eng, vol. 23, (1999), pp. 319-330.

[3] F. K. Scharfer and M. Herrwerth, "Shape Effects in Hypervelocity Impact on Semi Infinite Metallic Targets”, Int. J. Impact Eng, vol. 26, (2001), pp. 699-711.

[4] K. F. Hu and W. P. Schonberg, "Ballistic Limit Curves for Non- Spherical Projectiles Impacting Dual Wall Spacecraft Systems”, Int. J. Impact Eng, vol. 29, (2003), pp. 345-355.

[5] J. E. Williamsen and S. Evans, "Predicting orbital debris shape and orientation effects on spacecraft shield ballistic limits based on characteristic length", Int. J. Impact Eng, vol. 33, (2006), pp. 862-871.

[6] E. Corvonato, R. Destefanis and M. Faraud, "Integral Model for the Description of the Debris Cloud Structure and Impact", Int. J. Impact Eng, vol. 26, (2001), pp. 115-128. 